\title{
Novelty detection and memory processes within the human hippocampus Thomas Grunwald
}

\author{
Address: Dept. of Clinical Neurophysiology, Zurich Swiss Epilepsy Centre Zurich, Switzerland \\ from International Society on Brain and Behaviour: 3rd International Congress on Brain and Behaviour \\ Thessaloniki, Greece. 28 November - 2 December 2007 \\ Published: 17 April 2008 \\ Annals of General Psychiatry 2008, 7(Suppl I):S9I doi:I0.II86/I744-859X-7-SI-S9I
}

This abstract is available from: http://www.annals-general-psychiatry.com/content/7/SI/S9I

(C) 2008 Grunwald; licensee BioMed Central Ltd.

Intracranial recordings of cognitive potentials within the human hippocampal system have identified N400 potentials in the anterior medial temporal lobe (AMTL-N400), which are associated with successful encoding for declarative memory. In addition, their amplitudes to "new" but not "old" words in a verbal recognition task correlate both with verbal memory performance and with the neuronal density of the hippocampal CA1-region. Moreover, only AMTL-N400s to "new" but not those to "old" words can be reduced in amplitude by the NMDA-receptor blocker ketamine. Together, our findings indicate that successful encoding for declarative memory is mediated, at least in part, by NMDA-receptor dependent novelty detection within the human hippocampal system. However, the hippocampus proper is also known to participate in recall from episodic memory. Accordingly, only within the hippocampus proper do the neural responses to repeated words differ not only from those to new words but also from each other as a function of recognition success. By contrast parahippocampal (rhinal) responses are sensitive to repetition independent of conscious recognition. We take this finding to suggest that the hippocampus proper is specifically engaged during conscious memory processes. 